\title{
Genome-Wide Association Study Links Receptor Tyrosine Kinase Inhibitor Sprouty 2 to Thrombocytopenia after Coronary Artery Bypass Surgery
}

\author{
Jörn A. Karhausen ${ }^{1, *}$ Wenjing Qi ${ }^{2, *} \quad$ Alan M. Smeltz ${ }^{1} \quad$ Yi-Ju Li ${ }^{2,3} \quad$ Svati H. Shah ${ }^{3,4}$ William E. Kraus ${ }^{3,4}$ \\ Joseph P. Mathew ${ }^{1}$ Mihai V. Podgoreanu ${ }^{1, *}$ Miklos D. Kertai ${ }^{1,5, *}$ \\ for the Duke Perioperative Genetics Safety Outcomes (PEGASUS) Investigative Team**
}

\footnotetext{
1 Department of Anesthesiology, Duke Perioperative Genomics Program, Duke University Medical Center, Duke University, Durham, North Carolina, United States

2 Department of Biostatistics and Bioinformatics, Duke University Medical Center, Duke University, Durham, North Carolina, United States

${ }_{3}$ Molecular Physiology Institute, Duke University Medical Center, Duke University, Durham, North Carolina, United States

${ }^{4}$ Division of Cardiology, Department of Medicine, Duke University Medical

Center, Duke University, Durham, North Carolina, United States

${ }^{5}$ Department of Anesthesiology, Vanderbilt University Medical

Center, Vanderbilt University, Nashville, Tennessee, United States
}

Address for correspondence Jörn A. Karhausen, MD, Department of Anesthesiology, Duke University Medical Center, DUMC 3094, 2301 Erwin Road, 5693 HAFS Building, Durham, NC 27710, United States (e-mail: jorn.karhausen@duke.edu).

Thromb Haemost 2018;118:1572-1585.

\begin{abstract}
Introduction Thrombocytopenia after cardiac surgery independently predicts stroke, acute kidney injury and death. To understand the underlying risks and mechanisms, we analysed genetic variations associated with thrombocytopenia in patients undergoing coronary artery bypass grafting (CABG) surgery.

Materials and Methods Study subjects underwent isolated on-pump CABG surgery at Duke University Medical Center. Post-operative thrombocytopenia was defined as platelet count $<100 \times 10^{9} / \mathrm{L}$. Using a logistic regression model adjusted for clinical risk factors, we performed a genome-wide association study in a discovery cohort $(n=860)$ and validated significant findings in a replication cohort $(n=296)$. Protein expression was assessed in isolated platelets by immunoblot.

Keywords

- extracorporal circulation

- inherited/acquired platelet disorders

- gene mutations

Results A total of 63 single-nucleotide polymorphisms met a priori discovery thresholds for replication, but only 1 (rs9574547) in the intergenic region upstream of sprouty 2 (SPRY2) met nominal significance in the replication cohort. The minor allele of rs 9574547 was associated with a lower risk for thrombocytopenia (discovery cohort, odds ratio, $0.45,95 \%$ confidence interval, $0.30-0.67, p=9.76 \times 10^{-5}$ ) with the overall association confirmed by meta-analysis (meta- $p=7.88 \times 10^{-6}$ ). Immunoblotting demonstrated expression of
\end{abstract}

* The first two and last two authors contributed equally to this work.

** Members of the Duke Perioperative Genetics and Safety Outcomes (PEGASUS) Investigative Team are acknowledged in the Acknowledgment section. received

December 16, 2017

accepted after revision

June 8, 2018 (c) 2018 Georg Thieme Verlag KG Stuttgart - New York
DOI https://doi.org/

10.1055/s-0038-1667199. ISSN 0340-6245. 
SPRY2 and its dynamic regulation during platelet activation. Treatment with a functional SPRY2 peptide blunted platelet extracellular signal-regulated kinase (ERK) phosphorylation after agonist stimulation.

Conclusion We identified the association of a genetic polymorphism in the intergenic region of SPRY2 with a decreased incidence of thrombocytopenia after CABG surgery. Because SPRY2-an endogenous receptor tyrosine kinase inhibitor-is present in platelets and modulates essential signalling pathways, these findings support a role for SPRY2 as a novel modulator of platelet responses after cardiac surgery.

\section{Introduction}

Coronary artery bypass grafting (CABG) surgery with cardiopulmonary bypass ( $\mathrm{CPB}$ ) stimulates powerful inflammatory and tissue-injurious responses that can lead to significant organ damage-including acute kidney, neurocognitive and lung injury-and cause considerable morbidity and mortality. ${ }^{1}$ However, the mechanisms that drive tissue injury in this setting are not well defined. This deficit is a major obstacle to the development of effective preventive and treatment strategies.

We recently reported that, similar to observations in critically ill patients, ${ }^{2}$ post-operative thrombocytopenia (i.e. a minimum in-hospital platelet value of $<100 \times 10^{9}$ / $\mathrm{L})$ is associated with acute kidney injury (AKI), stroke and increased risk for mortality after CABG surgery. ${ }^{3,4}$ Indeed, cardiac surgery is associated with dramatic acute changes in platelet function that manifest both peri-operatively, as platelet dysfunction, and post-operatively, as platelet hyper-reactivity. ${ }^{5}$

Platelets have emerged as important and ubiquitous regulators of systemic and local inflammation with powerful influences on endothelial responses, neutrophil recruitment and associated distant-organ injury. ${ }^{6,7}$ Observations of organprotective effects of peri-operative anti-platelet therapy in cardiac surgery patients, ${ }^{8,9}$ and the association of thrombocytopenia with post-operative platelet responsiveness (evidenced by the increased incidence of ischaemic stroke) in our earlier study, ${ }^{4}$ strongly suggest that platelet activation and resultant consumption lead to the reduction of circulating platelet numbers. In this setting, end-organ damage could be mediated through small vessel occlusion and/or plateletdependent micro-vascular inflammation, attributing to platelets a much more central role within the pathophysiology of peri-operative organ injury than currently thought. Together with an increasing appreciation of platelets as important inflammatory cells such data could indicate the need for a re-evaluation of current anticoagulation therapies. However, since risk for bleeding is also associated with peri-operative anti-platelet therapy, it is crucial to first define the aetiology of thrombocytopenia after CABG surgery and its link to the development of adverse outcomes.

Genetic association studies are powerful tools for identifying disease-associated genes and for discovering previously unidentified pathways that contribute to certain phenotypes. Many of these studies have demonstrated that genetic variants play a substantial role in altering platelet function. ${ }^{10-15}$ Such variants, or single-nucleotide polymorphisms (SNPs), have been reported in genes that regulate key surface receptors and reactive granule constituents. ${ }^{10,12}$ More recently, however, SNPs have been found in non-coding regions associated with enhancer elements or promoters in megakaryocytes. ${ }^{11}$ In contrast to these large-scale, community-based cohort studies, a few, largely candidate-gene, studies have searched for genetic variants that influence acute platelet responses to cardiac surgery and CPB, and have identified gene polymorphisms that are associated with peri-operative bleeding ${ }^{16,17}$ and risk for post-operative cardiac injury. ${ }^{18,19}$

Since currently known clinical and procedural risk factors $^{3,4,8}$ do not adequately account for variability in the occurrence of post-operative thrombocytopenia, we hypothesized that within a multifactorial aetiology, genetic variations play a significant role. To test this hypothesis and to learn more about relevant platelet regulatory pathways, we conducted a genome-wide association study (GWAS) aimed at identifying common genetic variants associated with post-operative thrombocytopenia in the setting of CABG surgery.

\section{Materials and Methods}

We designed this study and reported our findings according to the 'Strengthening the Reporting of Genetic Association Studies' recommendations. ${ }^{20}$ Two independent cohorts of patients who underwent CABG surgery at the Duke Heart Center at Duke University Medical Center, Durham, North Carolina, United States, were used for initial common variant discovery by GWAS and replication analysis of top candidate SNPs. Each of the parent studies was approved by the Institutional Review Board at Duke University Medical Center and all subjects provided written informed consent.

Our discovery cohort was composed of a 1,004-patient sub-set from the Perioperative Genetics and Safety Outcomes Study (PEGASUS), comprising prospectively enrolled patients who underwent isolated non-emergent CABG surgery with $\mathrm{CPB}$ between 1997 and 2006. ${ }^{21}$ For patients who had more than one cardiac surgery during that period, only data from the first surgery were included. Of the original 1,004 study subjects, 860 patients met our inclusion criteria 
of self-reported European ancestry and complete phenotypic and genotypic data.

Our replication cohort was composed of patients in the CATHeterization GENetics (CATHGEN) study who underwent cardiac catheterization between 2001 and 2010 to evaluate ischaemic heart disease, and who subsequently underwent CABG surgery with CPB between 2006 and 2010. Of the 475 patients reviewed, 296 without concurrent valve surgery met our inclusion criteria.

Intra-operative anaesthetic, perfusion, cardioprotective and transfusion management was standardized, as described previously. ${ }^{21,22}$ Briefly, general anaesthesia was maintained with a combination of fentanyl and isoflurane. Perfusion support consisted of non-pulsatile $\mathrm{CPB}\left(30^{\circ} \mathrm{C}-32^{\circ} \mathrm{C}\right)$, crystalloid prime, pump flow rates $>2.4 \mathrm{~L} / \mathrm{min}$ per $\mathrm{m}^{2}$, cold blood cardioplegia, $\alpha$-stat blood-gas management, activated clotting times $>450$ seconds maintained with heparin, $\varepsilon$-aminocaproic acid infusion administered routinely and serial haematocrits maintained at $>0.18$. During the study period, the decision for intra-operative and post-operative blood and blood product transfusion was guided by laboratory testing consistent with the recommendations of the American Society of Anesthesiologists in 'Practice Guidelines for Blood Component Therapy, ${ }^{23}$

\section{Clinical Risk Factors, Data Collection and Endpoint Definition}

Patient and procedural characteristics for both cohorts were recorded and collected using the Duke Information System for Cardiovascular Care, an integral part of the Duke Databank for Cardiovascular Disease. The clinical risk factors for post-operative thrombocytopenia included patient characteristics, pre-operative cardiovascular medication use, CPB and aortic cross-clamp times, insertion of intra-aortic balloon pump, intra-operative and/or post-operative blood or blood product transfusions and platelet counts. Per institutional practice, pre-operative anti-platelet therapy with aspirin was maintained until the day before surgery; clopidogrel was discontinued at least 7 days before surgery; and warfarin was discontinued 4 days before surgery and 'bridged' with intravenous heparin infusion. ${ }^{3}$

Also per institutional practice, platelet counts for both the discovery and replication cohorts were measured in the Duke Clinical Pathology Laboratory on a pocH-100i automated hematology analyzer (Sysmex, Kobe, Japan) at baseline (pre-operative) and daily post-operatively until postoperative day 10 or at discharge, whichever came first. Postoperative thrombocytopenia as a qualitative endophenotype was the primary outcome of the study. This outcome was ascertained by selecting subjects whose post-operative minimum (nadir) platelet values were $<100 \times 10^{9} / \mathrm{L}($ moderate to severe thrombocytopenia group) and comparing to subjects whose post-operative minimum (nadir) platelet values were $>150 \times 10^{9} / \mathrm{L}$ (normal post-operative platelet count group). Further, since a continuous outcome is known to be generally more informative than a dichotomous outcome, we also used minimum (nadir) post-operative platelet values as a quantitative trait of thrombocytopenia (second- ary outcome of the study) to enhance the power of our study in detecting potential associations between genetic risk variants and post-operative platelet count.

\section{Genotyping and Quality Controls}

Genotyping platforms and quality controls (QCs) for genotype and sample exclusion have been described previously. ${ }^{21}$ Briefly, all of the 1,004 samples in the PEGASUS cohort (discovery samples) were genotyped at the Duke Genomic Analysis Facility using the Illumina Human610-Quad BeadChip (Illumina, Inc., San Diego, California, United States). Human genome build 37 (GRCh37/hg19) from February 2009 was used for the reference of ranges of genes and locations of SNPs. Various QC criteria were applied to ensure genotype quality. Markers with a GenCall (http://support.illumina.com/ downloads/gencall_software.html) score of $\leq 0.15$ or call frequency of $<98 \%$ were excluded. Samples with a call rate of $<98 \%$ or sex specification errors were also excluded in this initial QC. At the sample level, we used the PLINK software (https://www.cog-genomics.org/plink2) to check cryptic relatedness and duplications. ${ }^{24}$ For a pair of samples with an identity-by-descent value $>0.1875$ (between second- and third-degree relative), one sample was excluded from further analysis. Population structure was investigated using the EigenSoft program ${ }^{25}$ to generate 15 principal components (PCs) and multiple PC plots to identify any obvious outliers that deviated from the main cluster and, hence, should be excluded. As expected, we found no PCs that were associated with post-operative thrombocytopenia. Consequently, no PCs were included in the final association analysis models. At this stage, the QCed genotype dataset consisted of 960 study subjects with 561,091 markers. However, 100 of these patients were excluded from further analysis because of interventions in addition to their CABG surgery (e.g. combined CABG/valve surgery). Thus, the final discovery dataset (PEGASUS cohort) consisted of 860 patients of European descent with both genotype and phenotype data available.

All CATHGEN samples were genotyped at the Duke Genomic Analysis Facility using the Illumina OMNI 1-Quad BeadChip (Illumina, Inc.), and were subject to the same marker and sample QC criteria as described above for PEGASUS. GRCh37/hg19 was also used for reference for CATHGEN samples. For the replication dataset, we selected a sub-set of 296 patients out of 475 subjects from the CATHGEN cohort based on availability of genotype and patient and procedural characteristics.

Demographic and clinical characteristics, as well as comparisons between the two cohorts, are shown in -Supplementary Table S1 (available in the online version). In the discovery cohort, the primary outcome-moderate to severe post-operative thrombocytopenia-was observed in 176 out of 860 (20\%) patients (post-operative minimum [nadir] platelet value $<100 \times 10^{9} / \mathrm{L}$ ), while 268 out of 860 (31\%) patients had a normal post-operative platelet count (post-operative minimum [nadir] platelet value $>150$ $\left.\times 10^{9} / \mathrm{L}\right)$. In the replication cohort, 76 out of $296(26 \%)$ patients developed moderate to severe post-operative 
thrombocytopenia, while 70 out of 296 (24\%) patients had a normal post-operative platelet count. Only genotyped SNPs identified in the discovery cohort were tested in the CATHGEN cohort for replication purposes. Since different genotype platforms were used for the discovery and replication samples, we used imputed markers in the replication cohort to maximize the shared SNPs between the two cohorts. We also imputed markers for the purpose of fine mapping in the genetic regions of interest in the discovery cohort. To impute untyped SNPs, we used the IMPUTE2 program $^{26}$ and the post-QCed PEGASUS genotype cohort (960 samples with 561,091 markers) and we phased haplotypes from the 1,000 genome European (EUR) reference panel.

\section{Ex Vivo Platelet Analysis}

Whole blood $(40 \mathrm{~mL})$ was collected in acid citrate dextrose (ACD) sodium citrate (1:9 ACD v/v) from healthy individuals under a protocol approved by the Institutional Review Board at Duke University Medical Center. Blood was centrifuged at $120 \times \mathrm{g}$ for 8 minutes to isolate platelet-rich plasma (PRP). Platelets were isolated from the PRP by spinning at $650 \times g$ and washed in buffer containing $36 \mathrm{mM}$ citric acid, $5 \mathrm{mM}$ glucose, $5 \mathrm{mM} \mathrm{KCl}, 1 \mathrm{mM} \mathrm{MgCl}_{2}, 103 \mathrm{mM} \mathrm{NaCl}, 2 \mathrm{mM} \mathrm{CaCl}_{2}$, $3.5 \mathrm{~g} / \mathrm{L}$ bovine serum albumin and re-suspended at a concentration of $1 \mathrm{E}+07 / \mathrm{mL}$ in standard Tyrode's buffer. During entire isolation, platelet activation was prevented by addition of $1 \mu \mathrm{M}$ prostaglandin E1 (PGE1) and $0.2 \mathrm{U} / \mathrm{mL}$ apyrase (Millipore Sigma, Burlington, Massachusetts, United States). In a sub-set of experiments, the washed platelets were passed through a neutrophil reduction filter (Pall Corporation, Port Washington, New York, United States) to eliminate contamination of the platelet population.

Final platelet populations were then immediately harvested (resting) or activated for 2, 5, 15 or 30 minutes using thrombin at concentrations as outlined (Millipore Sigma), 60 $\mathrm{ng} / \mathrm{mL}$ convulxin (Cayman Chemical, Ann Arbor, Michigan, United States), or a mixture of $100 \mu \mathrm{M}$ adenosine diphosphate (ADP) (Bio/Data, Horsham, Pennsylvania, United States) and $100 \mu \mathrm{M}$ epinephrine (Millipore Sigma). At each activation time point, a platelet aliquot was fixed for $10 \mathrm{~min}$ utes in $1 \%$ formalin and the remaining platelets were lysed in their reaction buffer by addition of sodium dodecyl sulfate (SDS) to a final concentration of $2 \%$.

The fixed platelets were used to document the level of platelet activation at the time points examined by western blotting. For this, platelets were re-suspended in Phosphatebuffered saline, stained with anti-CD62P-APC antibody (BD Biosciences, Franklin Lakes, New Jersey, United States) and analysed immediately on a FACSCalibur Flow cytometer. Results were based on the analysis of 150,000 events/sample. Isotype control antibody-treated (BD Biosciences) samples were used as negative controls.

Western blotting was performed after determining and normalizing the protein content in the lysates (DC protein assay, BioRad, Hercules, California, United States). After adding $4 \times$ Laemmli buffer (BioRad) and boiling, samples were separated by SDS-polyacrylamide gel electrophoresis on $12 \%$ gradient gels and transferred to polyvinylidene difluoride membranes (BioRad). Membranes were blocked using Tris/HCl-buffered salt solution supplemented with $0.1 \%$ Tween 20 and $5 \%$ skim milk powder, and then incubated with primary antibodies overnight at $4^{\circ} \mathrm{C}$. Primary antibodies were anti-Sprouty-2 (Abcam, Cambridge, Massachusetts, United States) and anti-CD45 (Thermo Fisher Scientific, Waltham, Massachusetts, United States), antiextracellular signal-regulated kinase (ERK) $1 / 2$ (Cell Signaling Technology, Danvers, Massachusetts, United States), anti-pERK (Santa Cruz Biotechnology, Dallas, Texas, United States) and anti-phosphotyrosine (Cell Signaling). After washing, membranes were incubated with anti-rabbit or anti-mouse horseradish peroxidase conjugates (BioRad) and targets were visualized using Supersignal West Pico Luminol Enhancer solution (Thermo Fisher). Membranes were then stripped and re-probed with a mouse monoclonal anti- $\beta$-actin antibody (Millipore Sigma) as loading control.

For phosphatase treatment, activated platelets were lysed in buffer containing $10 \mathrm{mM}$ TRIS, $10 \mathrm{mM} \mathrm{NaCl}$ and 1\% NP40LB and aliquoted to control treatment, treatment with calf alkaline phosphatase or lambda phosphatase (both Millipore Sigma). Reactions were stopped by adding $1 \%$ SDS and boiling for 10 minutes.

SPRY2 octapeptides were custom synthesized by peptide 2.0 (Chantilly, Virginia, United States) based on protein sequences published by Hanafusa et al. ${ }^{27}$ To make peptides cell permeable, we added a c-terminal human immunodeficiency virus (HIV)-tat sequence as previously published. ${ }^{28}$ The final sequences were YGRKKRRQRRRTNEYTEGP-NH2 for the control peptide and YGRKKRRQRRRTNE(pY)TEGP-NH2 for the phosphorylated peptide. Peptides were solubilized as $50 \mathrm{mM}$ stock solutions in dimethyl sulfoxide. Washed platelets were treated with control on phosphorylated peptide at a final concentration of $50 \mu \mathrm{M}$ for 20 minutes in presence of apyrase and PGE1 as outlined above, then spun down and resuspended in Tyrode's buffer.

After this treatment, platelets remained in resting state as documented by lack of CD62P externalization and the lack of binding of Oregon488-labelled soluble fibrinogen (Thermo Fisher) (-Supplementary Fig. S1B, available in the online version). However, platelet remained functionally intact, as ascertained after platelet reconstitution in platelet-poor plasma and agonist-induced platelet aggregation using the PAP-8E Platelet Aggregation Profiler (Bio/Data) (-Supplementary Fig. S1A, available in the online version).

\section{Statistical Analysis}

Descriptive statistics of clinical variables are presented as frequency and percentage for categorical variables and mean \pm standard deviation (SD) for continuous variables. Univariable and multivariable logistic regression models were applied to evaluate the association between demographic, clinical and procedural characteristics with moderate to severe post-operative thrombocytopenia (minimum [nadir] platelet values $<100 \times 10^{9} / \mathrm{L}$ ). To derive the final multivariable logistic regression model containing variables with $p$ values of $<0.05$, univariable associations with a $p$-value of $<0.15$ were evaluated using a backward stepwise 
technique and the Schwarz-Bayes criterion. ${ }^{29}$ Analyses of demographic, clinical and procedural characteristics were conducted using SAS version 9.4 (SAS Institute, Inc., Cary, North Carolina, United States).

Genetic association analyses were performed using PLINK (https://www.cog-genomics.org/plink2) for all genotyped markers in the discovery cohort. At the data analysis stage, additional QC criteria excluded markers that were significantly deviated from the Hardy-Weinberg equilibrium $\left(p<10^{-6}\right)$ or had a minor allele frequency (MAF) $<2 \%$. For each of the SNPs, allelic associations with post-operative thrombocytopenia (defined as a qualitative trait [primary outcome analysis] or as a quantitative trait [secondary outcome analysis]) were assessed using multivariable logistic regression analyses or multivariable linear regression analyses as appropriate, adjusted for the same set of clinical variables identified as part of the final multivariable logistic regression model containing demographic, clinical and procedural characteristics. These association tests assumed an additive inheritance model (homozygote major allele vs. heterozygote vs. homozygote minor allele). Markers with $p<5 \times 10^{-8}$ were considered to be genome-wide significant, which is the most commonly accepted significance threshold for GWAS. In addition to this stringent criterion, a relaxed significance threshold of $p<10^{-4}$ was applied when choosing SNPs for replication in the CATHGEN cohort. $^{22}$ Similar to the discovery cohort, 41 genotyped markers were tested using PLINK in the replication cohort. For 22 imputed markers in the discovery cohort, SNPTEST (https://mathgen.stats.ox.ac.uk/genetics_software/snptest/ snptest.html) was used to conduct genetic association analyses based on genotype dosages. The same multivariable logistic regression model adjusted for the final set of demographic, clinical and procedural characteristics as in the discovery cohort was applied to the replication cohort. To assess the overall effect of candidate SNPs, we conducted a meta-analysis as implemented in METAL (http://www.sph. umich.edu/csg/abecasis/metal).

The final candidate $\operatorname{SNP}(\mathrm{s})$ were prioritized based on (1) meeting nominal significance in the replication cohort, (2) showing the effect in the same direction for post-operative thrombocytopenia (defined as a qualitative trait) in the replication cohort as in the discovery cohort and (3) reaching statistical significance in the meta-analysis. To further investigate whether clinical factors chosen by multivariable regression influenced the effect of the candidate SNPs on the primary outcome, moderate to severe post-operative thrombocytopenia, we also tested the regression models on the primary outcome adjusted for the significant clinical factors (age, duration of CPB and use of blood products) and the interaction between these clinical factors and the identified candidate SNPs.

Marker density for the top candidate gene(s) or region(s) was increased by using imputed markers within the gene or region in the discovery cohort to examine the association pattern within the region. SNPTEST was again used to conduct genetic association analyses based on genotype dosages for imputed markers. Markers with info measure (measure of the observed statistical information associated with the allele frequency estimate) below 0.4 were excluded from the analyses. Regional association plots of genome-wide association results within the region of interest were generated using LocusZoom. ${ }^{30}$ Finally, we used genome-wide complex trait analysis (http://cnsgenomics.com/software/gcta/) to estimate the extent of variance in the primary outcome (moderate to severe post-operative thrombocytopenia as a qualitative trait) that was attributed to candidate SNPs.

\section{Results}

For our study, the primary outcome-moderate to severe postoperative thrombocytopenia-was present in 176 patients in the discovery dataset and 76 patients in the replication dataset. Demographic, clinical and procedural characteristics of the patients in these two datasets, stratified according to moderate to severe post-operative thrombocytopenia or normal postoperative platelet count, are shown in -Table 1 . The overall mean age was $63 \pm 11$ years in the discovery dataset and $61 \pm 11$ years in the replication dataset. Both datasets had higher proportions of male patients (340 [76.6\%] in the discovery cohort and 100 [68.5\%] in the replication cohort). The mean \pm SD post-operative minimum (nadir) platelet counts was similar in the two datasets $\left(145 \pm 60\right.$ vs. $\left.131 \pm 59 \times 10^{9} / \mathrm{L}\right)$. In the discovery dataset, patients with moderate to severe post-operative thrombocytopenia had a significantly lower mean post-operative minimum (nadir) platelet count compared with controls with normal post-operative platelet count $\left(81 \times 10^{9} / \mathrm{L}[ \pm 16]\right.$ vs. $\left.187 \times 10^{9} / \mathrm{L}[ \pm 37], p<0.0001\right)$. Similarly, patients in the replication dataset with moderate to severe post-operative thrombocytopenia had a significantly lower mean post-operative minimum (nadir) platelet count compared with controls with normal post-operative platelet count $\left(79 \times 10^{9} / \mathrm{L}[ \pm 15]\right.$ vs. $\left.187 \times 10^{9} / \mathrm{L}[ \pm 29], p<0.0001\right)$.

Univariable predictors of moderate to severe post-operative thrombocytopenia are shown in -Supplementary Table S2 (available in the online version). Several demographic, clinical and procedural characteristics were significantly associated with increased risk for moderate to severe post-operative thrombocytopenia. According to our multivariable analysis, age, duration of CPB and use of blood products intra-operatively and/or within 2 days post-operatively remained independent risk factors for moderate to severe post-operative thrombocytopenia (-Supplementary Table S2, available in the online version), and were subsequently incorporated as covariates in multivariable logistic regression models to adjust the SNP associations with postoperative thrombocytopenia.

After applying our initial QCs, 561,091 genotyped markers were available for analysis. Of these, 3 SNPs were excluded due to deviation from the Hardy-Weinberg equilibrium, and 36,022 SNPs were excluded due to MAF $<0.02$. The remaining 525,066 markers were tested in the 444 subjects in the discovery dataset for association with the primary outcome of moderate to severe post-operative thrombocytopenia. GWAS results in the discovery cohort are depicted using a Manhattan plot (-Supplementary Fig. S2, available in the online version). None of the SNPs reached genome-wide 
Table 1 Demographic, clinical and procedural characteristics of discovery and replication datasets based on presence of moderate to severe postoperative thrombocytopenia

\begin{tabular}{|c|c|c|c|c|c|c|}
\hline \multirow{2}{*}{ Predictor } & \multicolumn{3}{|c|}{ Discovery dataset $(N=444)$} & \multicolumn{3}{|c|}{ Replication dataset $(N=146)$} \\
\hline & $\begin{array}{l}\text { Patients with } \\
\text { nadir platelet } \\
>150 \times 10^{9} / \mathrm{L} \\
(n=268)\end{array}$ & $\begin{array}{l}\text { Patients with } \\
\text { nadir platelet } \\
<100 \times 10^{9} / \mathrm{L} \\
(n=176)\end{array}$ & $p$-Value ${ }^{a}$ & $\begin{array}{l}\text { Patients with } \\
\text { nadir platelet } \\
>150 \times 10^{9} / \mathrm{L} \\
(n=70)\end{array}$ & $\begin{array}{l}\text { Patients with } \\
\text { nadir platelet } \\
<100 \times 10^{9} / \mathrm{L} \\
(n=76)\end{array}$ & $p$-Value ${ }^{a}$ \\
\hline \multicolumn{7}{|l|}{ Demographics } \\
\hline Age, y & $60.86 \pm 10.26$ & $66.93 \pm 9.82$ & $<0.0001$ & $56.10 \pm 10.82$ & $65.37 \pm 9.16$ & $<0.0001^{\mathrm{b}}$ \\
\hline Female sex & $62(23.13)$ & $42(23.86)$ & 0.859 & $16(22.86)$ & $30(39.47)$ & 0.031 \\
\hline \multicolumn{7}{|l|}{ Laboratory test result } \\
\hline Preoperative creatinine, $\mathrm{mg} / \mathrm{dL}$ & $1.0 \pm 0.23$ & $1.28 \pm 1.40$ & 0.0001 & $1.06 \pm 0.39$ & $1.08 \pm 0.33$ & 0.669 \\
\hline $\begin{array}{l}\text { Minimum postoperative plate- } \\
\text { let count, } 10^{9} / \mathrm{L}\end{array}$ & $187.40 \pm 36.73$ & $80.52 \pm 15.62$ & $<0.0001$ & $186.74 \pm 29.25$ & $78.82 \pm 15.16$ & $<0.0001$ \\
\hline \multicolumn{7}{|l|}{ Left ventricular function } \\
\hline Normal & $178(66.42)$ & $93(52.84)$ & \multirow[t]{3}{*}{0.0107} & $36(51.43)$ & $42(55.26)$ & \multirow[t]{3}{*}{0.399} \\
\hline Moderate dysfunction & $81(30.22)$ & $71(40.34)$ & & $30(42.86)$ & $26(34.21)$ & \\
\hline Severe dysfunction & $9(3.36)$ & $12(6.82)$ & & $4(5.71)$ & $8(10.53)$ & \\
\hline \multicolumn{7}{|l|}{ Preoperative medications } \\
\hline Acetylsalicylic acid & $164(65.60)$ & $111(69.38)$ & 0.428 & $41(62.12)$ & $57(80.28)$ & 0.019 \\
\hline $\begin{array}{l}\text { Angiotensin-converting enzyme } \\
\text { inhibitors }\end{array}$ & $134(50.0)$ & $105(59.66)$ & 0.046 & 45 (64.29) & $46(60.53)$ & 0.640 \\
\hline Beta-receptor blockers & $206(76.87)$ & $145(82.39)$ & 0.162 & $56(80.0)$ & $70(92.11)$ & 0.034 \\
\hline Calcium-channel blockers & $52(20.97)$ & $25(15.63)$ & 0.178 & $10(15.15)$ & $12(17.39)$ & 0.725 \\
\hline Diuretics & $53(21.37)$ & $44(27.50)$ & 0.156 & $16(24.24)$ & $21(29.58)$ & 0.482 \\
\hline Nitrates & $92(37.10)$ & $62(38.75)$ & 0.737 & $17(25.76)$ & $25(35.21)$ & 0.231 \\
\hline Statins & $162(60.45)$ & $123(69.89)$ & 0.042 & $47(67.14)$ & $51(67.11)$ & 0.996 \\
\hline \multicolumn{7}{|l|}{ Intraoperative characteristics } \\
\hline $\begin{array}{l}\text { Duration of cardiopulmonary } \\
\text { bypass per minutes }\end{array}$ & $108.29 \pm 30.34$ & $131.10 \pm 49.44$ & $<0.0001$ & $110.51 \pm 35.06$ & $132.54 \pm 47.47$ & 0.002 \\
\hline $\begin{array}{l}\text { Duration of aortic-cross clamp } \\
\text { per minutes }\end{array}$ & $60.79 \pm 21.74$ & $73.82 \pm 34.19$ & $<0.0001$ & $57.46 \pm 21.88$ & $72.49 \pm 27.95$ & $0.0004^{\mathrm{b}}$ \\
\hline $\begin{array}{l}\text { Intraoperative insertion of } \\
\text { intra-aortic balloon pump }\end{array}$ & $5(1.87)$ & $16(9.09)$ & 0.0005 & $4(5.71)$ & 15 (19.74) & $0.014^{\mathrm{b}}$ \\
\hline $\begin{array}{l}\text { Blood product use intraopera- } \\
\text { tively and postoperative within } \\
2 \text { days of surgery }\end{array}$ & $67(25.28)$ & $109(62.64)$ & $<0.0001$ & $27(38.57)$ & $51(67.11)$ & 0.0006 \\
\hline
\end{tabular}

Note: Continuous variables are presented as means \pm standard deviation, and categorical variables as number (\%).

${ }^{a}$ Comparisons were made using 2 sample tests to test the differences in demographic, clinical and procedural characteristics between subjects with and without moderate to severe postoperative thrombocytopenia, separately for discovery and replication datasets.

${ }^{\mathrm{b}} p$-Values were derived from the Wilcoxon rank sum tests or 2 sample $t$-tests for continuous variables, and Chi-Square tests or Fisher exact tests for categorical variables, as appropriate.

significance. However, 63 SNPs met the a priori-defined discovery threshold of $p<10^{-4}$ and were then analysed in the replication dataset ( - Supplementary Table S3, available in the online version).

Of the 63 SNPs analysed in the replication dataset (-Table 2), 22 SNPs had imputed genotypes due to differences between the two BeadChips used in these two datasets. We identified one SNP, rs9574547, with nominal significance $(p<0.05)$ in the replication dataset (-Table 2$)$. In both datasets, the minor allele of rs9574547 in the intergenic region between LOC729479 and Sprouty receptor tyrosine kinase (RTK) signalling antagonist 2 (SPRY2) was associated with a decreased incidence of moderate to severe post-operative thrombocytopenia (discovery dataset: odds ratio [OR], 0.45; $95 \%$ confidence interval $[\mathrm{CI}], 0.30-0.67 ; p=9.76 \times 10^{-5}$; and replication dataset: $\mathrm{OR}, 0.47 ; 95 \% \mathrm{CI}, 0.24-0.92 ; p=0.03)$. The meta-analysis of both cohorts by METAL showed that rs9574547 remained significantly associated with decreased risk for moderate to severe post-operative thrombocytopenia (meta-p $=7.88 \times 10^{-6}$; - Table 2). 


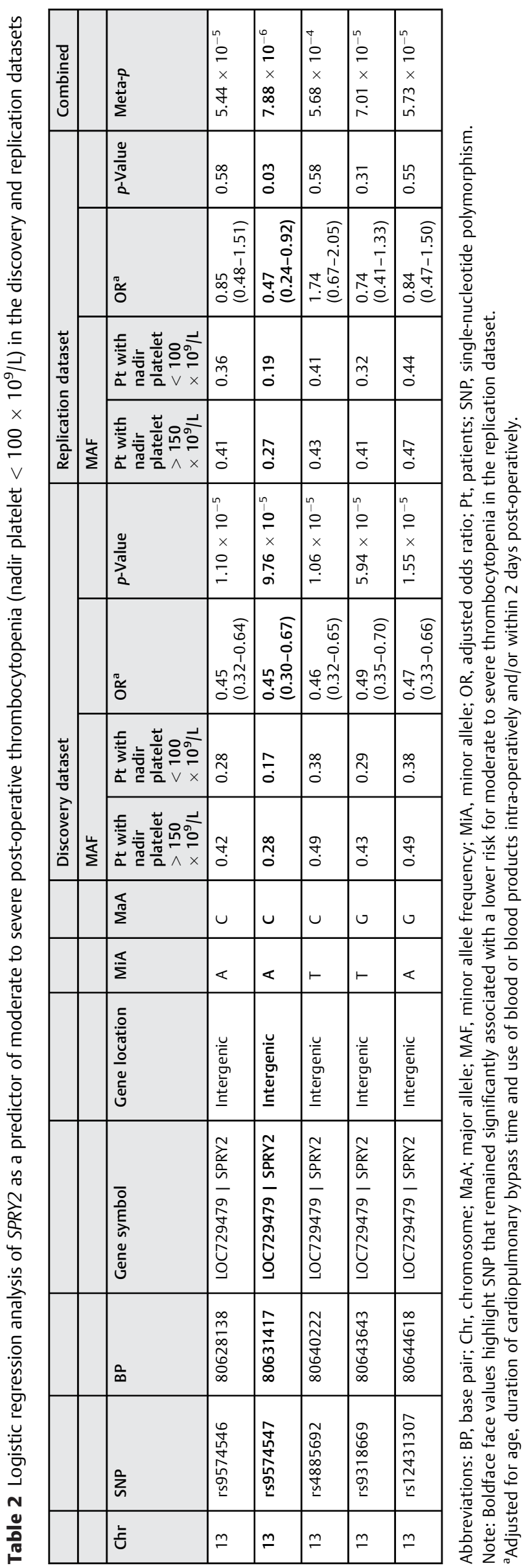

We further examined the regression models on moderate to severe post-operative thrombocytopenia adjusted for age, duration of CPB and use of blood products intra-operatively and/or within 2 days post-operatively and their interactions with rs9574547. None of the three interactions were statistically significant: interaction of age and rs9574547, $p=0.42$; interaction of duration of CPB time and rs9574547, $p=0.58$; interaction of blood transfusion and rs9574547, $p=0.30$.

Using a total of 1,353 imputed markers (info $\geq 0.4$ ), we increased the marker density in the LOC729479 to SPRY2 intergenic region on chromosome 13 to identify SNPs most strongly associated with moderate to severe post-operative thrombocytopenia and potentially close to the causal variants in this gene: 80585271-80915086 (in the LOC729479 to SPRY2 region). The relative location of the genotyped SNP rs9574547 in the LOC729479 I SPRY2 region, local linkage disequilibrium (LD) and recombination patterns are shown in -Fig. 1.

Finally, we performed genome-wide complex trait analyses to estimate the proportion of variance in moderate to severe post-operative thrombocytopenia explained by rs9574547. The results indicated that rs9574547 explained $3.41 \%$ of attributable variance in the risk of developing moderate to severe post-operative thrombocytopenia after CABG surgery.

To further study the association of identified SNPs with post-operative thrombocytopenia, defined as a quantitative thrombocytopenia trait, we conducted multivariable linear regression analyses adjusted for the same set of demographic, clinical and procedural variables as for the qualitative trait in the discovery $(N=860)$ and replication $(N=296)$ cohorts. The results showed significant association of the minor allele of rs9574547 in the LOC729479 I SPRY2 region with higher post-operative minimum platelet count in individual patients (for every additional minor allele of rs9574547, individual post-operative minimum platelet counts were estimated to increase by $10.8 \pm 2.5 ; p=1.62$ $\times 10^{-5}$ in the discovery cohort and by $10.2 \pm 3.8$, $p=0.0086$ in the replication cohort, -Supplementary Fig. S3, available in the online version).

We also studied changes in platelet counts throughout the peri-operative period in patients with and without postoperative thrombocytopenia. Our analysis revealed some notable differences in pre-operative platelet counts between patients in the moderate to severe post-operative thrombocytopenia group versus those in the normal post-operative platelet count group (-Fig. 2). This finding led us further to explore whether there was an association between rs9574547 and pre-operative platelet count. The result of our univariable analysis indicated that, indeed, there was a significant association between rs9574547 and pre-operative platelet count defined as a continuous variable $(p=0.004)$. Subsequently, we performed a multivariable linear regression analysis to study whether the association between rs9574547 and post-operative thrombocytopenia was independent from pre-operative platelet count and our results indicated that the association between rs9574547 and post-operative thrombocytopenia remained statistically significant $(p=0.0056)$. 


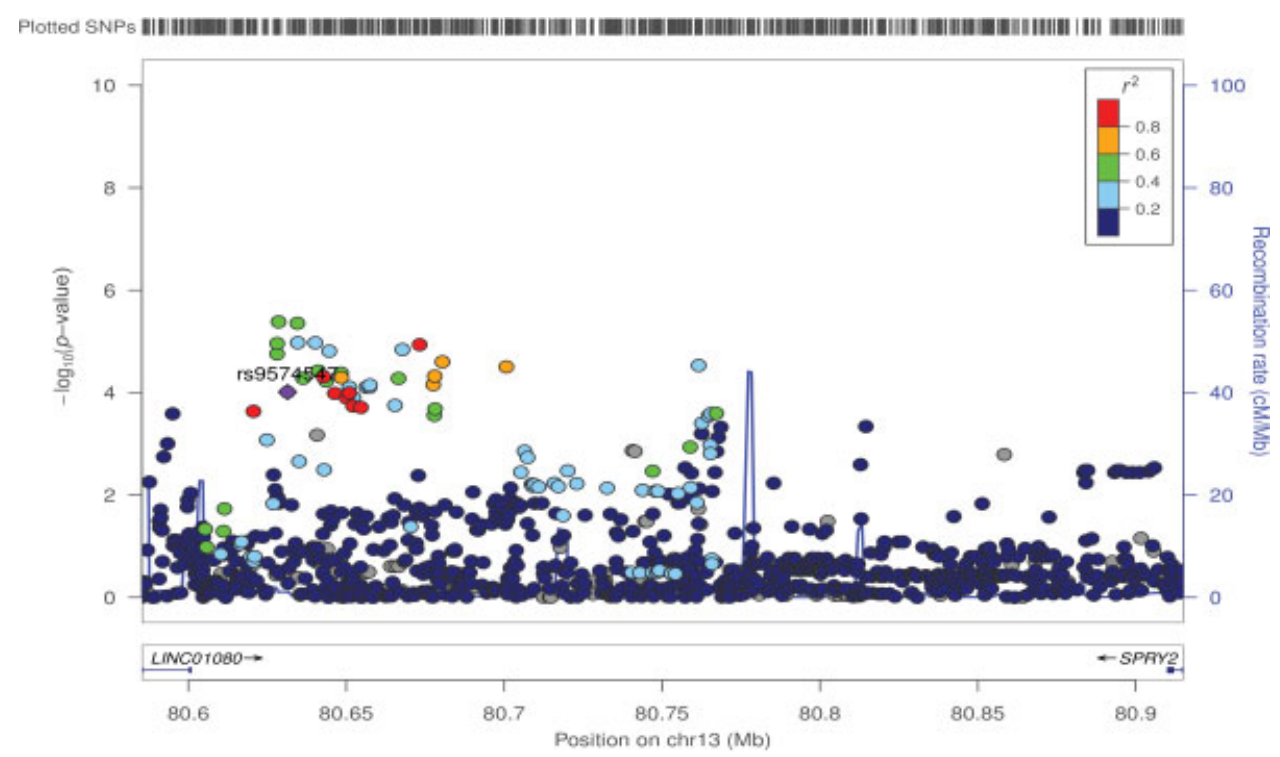

Fig. 1 LocusZoom plot of the LOC729479 I SPRY2 region based on the discovery cohort. The left $y$-axis is the - $\log _{10}$ ( $p$-value) of the tests of association and the right $y$-axis is the recombination rate (cM/Mb). The identified SNP, rs9574547, is plotted as the reference single-nucleotide polymorphism (SNP) and colour-coded as a purple diamond. Surrounding SNPs are plotted as circles colour-coded by strength of linkage disequilibrium (LD) measured as $r^{2}$, where red represents a complete or very strong $L D$, and blue represents a weak LD or independent variants. LD was calculated based on 1000 Genomes EUR genome build (November 2014).

\section{Ex Vivo Platelet Analysis}

To explore potential mechanisms behind the association of post-operative thrombocytopenia and the identified SNP, we next explored the possibility that SPRY2 is involved in regulating platelet reactivity. Because SPRY2 expression has not yet been documented in platelets, we first measured SPRY2 protein expression in isolated washed platelets before and after agonist stimulation. As expected, agonist treatment rapidly led to platelet activation as evidenced by surface expression of CD62P in fluorescence-activated cell sorting analysis (-Fig. 3A, - Supplementary Fig. 54, available in the online version). Robust SPRY2 protein expression was observed under baseline conditions as a single protein band. Following platelet activation either via the protease-activated

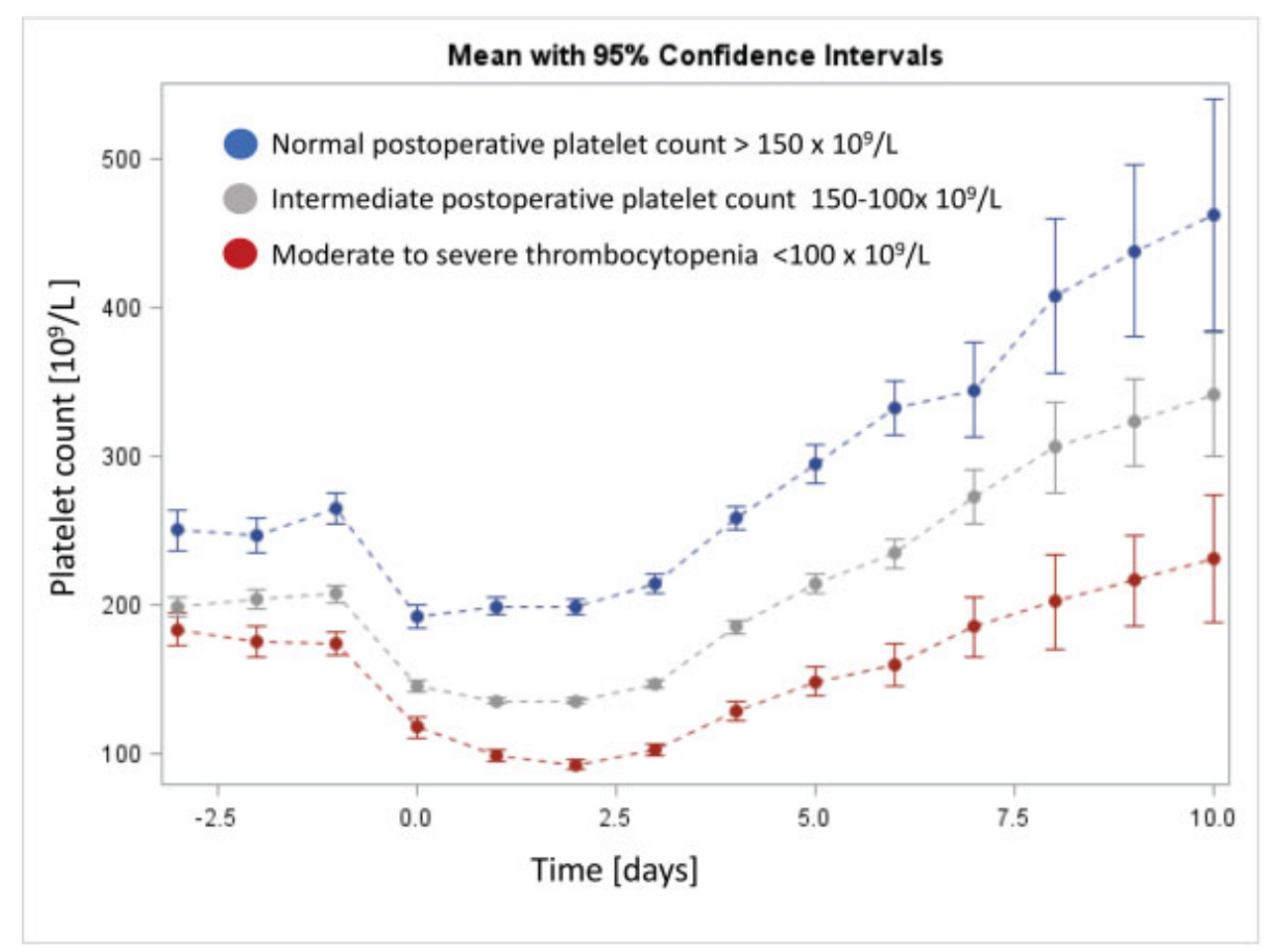

Fig. 2 Development of platelet counts in the peri-operative period. Platelet counts (in platelets $\times 10^{9} / \mathrm{L}$ ) were plotted during the peri-operative time period (day -3 to day +10 relative to the day of surgery) for the three groups defined by their nadir post-operative platelet count as noted in the legend. Values are shown as mean with $95 \%$ confidence intervals. 


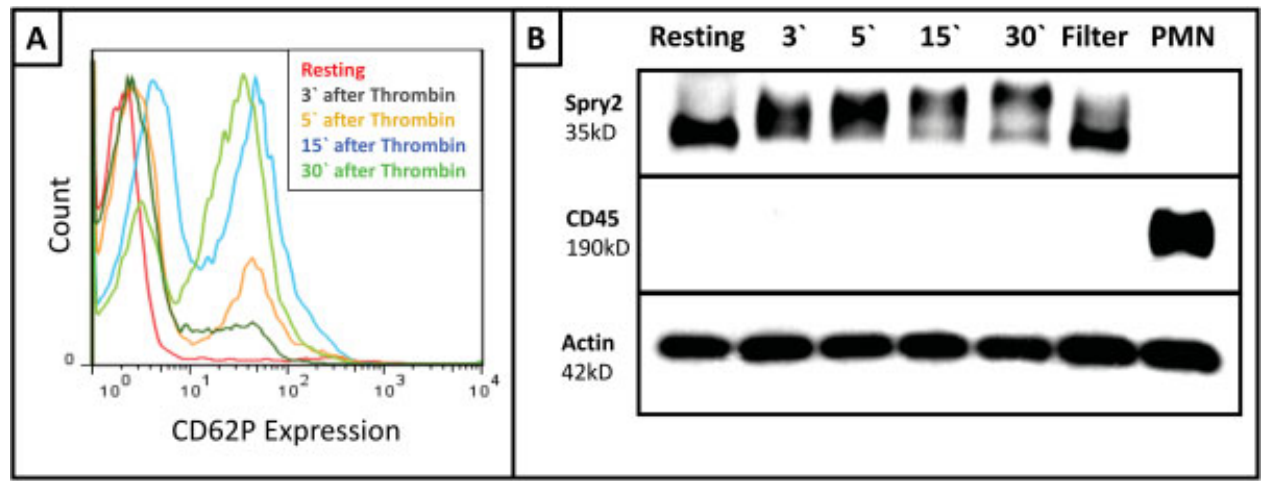

Fig. 3 Sprouty 2 is expressed in human platelets. Human platelets from healthy volunteers were isolated and activated with thrombin for indicated time points [in minutes]. (A) Flow cytometry was performed from aliquots of samples before platelet lysis and western blotting and surface expression of the platelet activation marker CD62P was measured. (B) Platelet proteins were separated by gel electrophoresis and probed using anti-Sprouty 2 antibody. Leukocyte contamination was excluded by passing a sub-set of unstimulated platelets through a leukocyte reduction filter ('Filter') and by probing for the leukocyte marker CD45 (isolated polymorphonuclear cells ['PMN'] were used to document positive CD45-staining). Beta-actin served as protein loading control. Protein size is denoted in kilo Dalton ( $k D)$. Images are representative of $n=5$ experiments from distinct volunteers.

receptor pathway using thrombin, via the glycoprotein VI (GPVI) receptor pathway using the collagen receptor agonist convulxin, or by co-stimulation of the purinergic P2Y12 and $\alpha$ (2A)-adrenergic receptor using epinephrine and ADP, however, a second higher molecular weight band appeared, and the initial lower molecular weight SPRY2 signal diminished (-Fig. 3B, -Supplementary Fig. S4, available in the online version). Overall SPRY2 protein levels did not change upon platelet activation, as quantified by densitometric measurements of the combined SPRY2 signal (data not shown). To verify purity, unstimulated platelets were passed through a leukocyte depletion filter, which led to some baseline platelet activation but supported our above observations. In addition, re-probing of blots for the pan-leukocyte epitope CD45 showed no indication for leukocyte contamination.

Because a crucial determinant of SPRY2 protein activity in cellular responses to growth factors is phosphorylation of its Tyr 55 residue, ${ }^{27}$ we next examined whether tyrosine phosphorylation may account for some of the change in the SPRY2 banding pattern after platelet activation. As shown in -Fig. 4A, we observed an increased phosphotyrosine band in the SPRY2 region by western blotting. In addition, phosphatase treatment of activated platelet lysate abolished the higher molecular weight band of SPRY2. Together, these

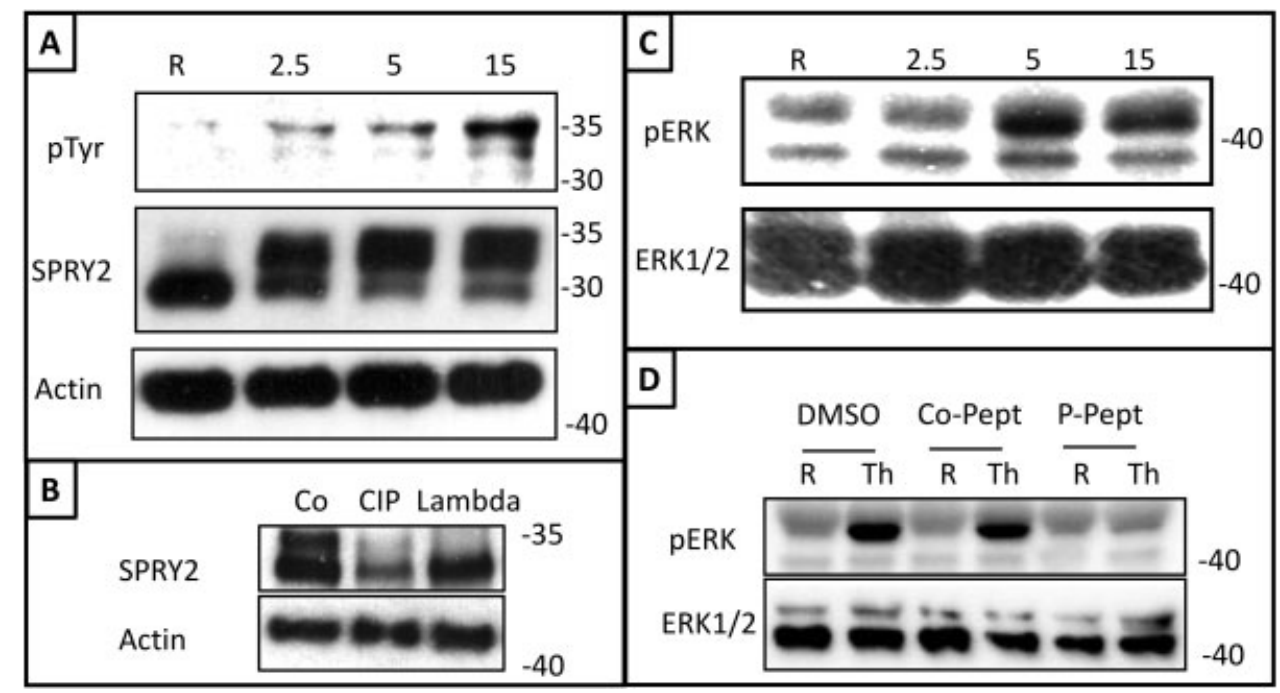

Fig. 4 Sprouty 2 modulates extracellular signal-regulated kinase (ERK) phosphorylation pathway during platelet activation. (A) Western blot analysis of platelet lysates following activation with $0.2 \mathrm{U} / \mathrm{mL}$ thrombin for indicated time points probed for phosphotyrosine (p-Tyr), Sprouty 2 (SPRY2) and $\beta$ - actin. (B) Platelet lysate from platelets activated for 5 minutes with $0.2 \mathrm{U} / \mathrm{mL}$ thrombin was treated with enzyme buffer (co), calf intestinal alkaline phosphatase (CIP) or lambda phosphatase. Western blot was labelled using anti-Sprouty2 (SPRY2) or anti- $\beta$ - actin antibodies. (C) Western blot analysis of platelet lysates following activation with $0.2 \mathrm{U} / \mathrm{ml}$ thrombin for indicated time points probed for ERK $1 / 2$ (ERK) or phospho-ERK (p-ERK). (D) Platelet were pre-treated with vehicle (dimethyl sulfoxide [DMSO]) or with $50 \mu \mathrm{M}$ of a Sprouty2-octapeptide either in its unphosphorylated (control: co-Pept) or phosphorylated form (P-Pept), exposed to $0.2 \mathrm{U} / \mathrm{mL}$ thrombin for 5 minutes and then lysed. Western blots were probed for ERK1/2 (ERK) or phospho-ERK (p-ERK). Molecular weight is indicated in kilo Dalton. Images are representative of three independent experiments. 
observations indicate that in the course of platelet activation, SPRY2 is modified by phosphorylation (-Fig. 4B).

Phosphorylation is essential for SPRY2 regulator functions of the mitogen-activated protein kinase (MAPK) pathway. ${ }^{27}$ Because the MAPK pathway is also activated during platelet stimulation ( - Fig. 4C), we next explored whether SPRY2 may have similar effects in platelet MAPK signalling using a previously published phospho-SPRY2 octapeptide ${ }^{27}$ that we made cell permeable through addition of a HIV-tat sequence. In accordance with the above report which had studied the effect of octapeptide micro-injections on fibroblast responses to growth factor treatment, ${ }^{27}$ pre-treatment of platelets with the phosphorylated SPRY2 peptide, but not with the non-phosphorylated control peptide, resulted in blunted phosphorylation of ERK after platelet stimulation (-Fig. 4D).

Therefore, our combined ex vivo data provide primary evidence that SPRY2 protein is present in circulating platelets and suggests that differential regulation of the SPRY2 protein during platelet activation may serve to dynamically modulate platelet functions.

\section{Discussion}

Using a GWAS approach, we identified a novel susceptibility locus at 13q31.1 (rs9574547) associated with moderate to severe post-operative thrombocytopenia after CABG surgery with $\mathrm{CPB}$. Patients carrying one or both of the minor alleles of this SNP were at decreased risk for moderate to severe post-operative thrombocytopenia. Notably, our findings suggested an independent association even after adjusting for clinical and surgery-related variables known to be associated with risk for post-operative thrombocytopenia. Corroborating our genome-wide association findings, we demonstrated for the first time that SPRY2 is expressed in resting platelets and provided evidence that SPRY2 may modify ERK phosphorylation in ex vivo platelet activation. These findings add to mounting data that implicate genetic variations in platelet responsiveness and post-operative thrombocytopenia. ${ }^{13,31,32}$ Importantly, we previously reported that a reduction in platelet count in patients undergoing cardiac surgery is linked to increased risk for AKI and mortality, and appears to occur in a state of platelet hyper-responsiveness, as evidenced by increased risk for stroke in these patients. ${ }^{3,4}$ Thus, understanding the role of SPRY2 in regulating platelet function may provide the key to defining the currently unresolved pathophysiology of postoperative thrombocytopenia after cardiac surgery and may lead to novel pharmacologic targets to prevent post-operative thrombocytopenia and subsequent complications after CABG surgery.

The 13q31.1 locus identified here is in the intergenic region bounded by LOC729479 (a hypothetical gene) and the SPRY2 gene (chr13q31.1; lowest meta- $p=7.9 \times 10^{-6}$ ). Out of these two genes, the protein encoded by SPRY2 serves as a crucial regulator of RTK signalling. Initially identified in Drosophila, SPRY is an endogenous inhibitor of growth factor signalling (mammalian SPRY2 and the single dSpry isoform found in Drosophila play similar roles reviewed in Guy et al ${ }^{33}$ ) that interferes with the assembly of essential adaptor protein complexes downstream of the RTK receptor. ${ }^{27}$ As such, SPRY2 functions in a classic feedback loop of RTK activity.

RTKs are a family of cell surface receptors that regulate key cellular processes including proliferation and differentiation, cell survival, metabolism, migration and cell cycle control. ${ }^{34}$ Although the function of RTK signalling has been most extensively examined in the context of effects on growth factors involved in proliferation and development, a critical influence on platelet activation has also been established. Most prominent is the collagen receptor GPVI, which is constitutively associated with an RTK, that is, the immune tyrosine activation motif (ITAM)-bearing Fc receptor $\gamma$-chain. ${ }^{35}$ Another example is the Tyro3/Axl/Mer (TAM) RTK family, which plays important roles in haemostasis and inflammation, for example, by interacting with vitamin K-dependent protein Gas6. ${ }^{36}$ Platelet activation via these receptors enables responses that are distinct from G-protein coupled receptor (GPCR) engagement, that is, after thrombin binding, ${ }^{35}$ and appear to allow very finely tuned and graded platelet responses. For example, ITAM signalling, but not GPCR signalling, controls vascular integrity during inflammation, ${ }^{37}$ and a deficiency in TAM receptor signalling protects against thrombosis but does not carry a bleeding phenotype. ${ }^{38}$

Thus, it is not surprising that, while RTK inhibitors in certain anti-cancer agents elicit significant platelet function abnormalities, ${ }^{39}$ tyrosine kinases are also increasingly recognized as potential targets for novel anti-platelet agents. ${ }^{40}$ The concept of such drugs is based on the notion that they may have the capacity to control the rheostat of platelet-inhibitory and platelet-activating factors, in contrast to currently approved drugs that cause profound platelet inhibition and therefore increase the risk for bleeding complications. Although many aspects of the tyrosine kinase signalling pathway in platelets have not yet been explored, our finding of a susceptibility locus for moderate to severe post-operative thrombocytopenia in the intergenic region upstream of the SPRY2 gene may be useful in the search for regulators of tyrosine kinases in platelets.

Importantly, while the role of SPRY2 has not been investigated in the context of platelet function, its major binding partners are strongly implicated in platelet regulation. For example, SPRY2 impedes interaction between adaptor protein growth factor receptor-bound protein 2 (Grb2) and RTKs, thereby acting as an endogenous RTK inhibitor. ${ }^{27}$ Importantly, Grb2 regulates collagen receptor signalling in platelets and, thus, also serves as an important modulator of haemostasis and thrombosis. ${ }^{41}$ Based on these reports, it would be expected that the endogenous RTK inhibitor SPRY2 functions in a platelet inhibitory way and thus leads to a similar platelet phenotype as Grb2 knockout, ${ }^{41}$ or RTK inhibitors used in anti-cancer therapy. ${ }^{39}$

Although SPRY2 has been indirectly associated with megakaryocytic differentiation, ${ }^{42}$ its expression in platelets has not been documented. This presented a major obstacle to assessing the relevance of our GWAS finding to possible platelet function abnormalities. Our finding that, indeed, 
SPRY2 is expressed in platelets and, further, that it appears to be post-translationally modified after platelet activation, provides important support for further investigation of SPRY2 as a modulator of platelet function. SPRY2 functions are tightly regulated by post-translational modification including phosphorylation, ${ }^{27}$ ubiquitination and proteasomal degradation. ${ }^{43,44}$ However, of note, our data, which supports changes in SPRY2 banding patterns in response to diverse platelet agonists, does not fully rule out pathway cross-activation and more detailed analyses are needed to define the exact signalling pathway that regulates SPRY2 responses.

To provide first insights into how SPRY2 might function in platelets, we adapted an approach previously used by Hanafusa et al. ${ }^{27}$ Importantly, evidence of SPRY2 tyrosine phosphorylation following platelet activation suggested that SPRY2 may function in a similar fashion, as noted by these authors when examining SPRY2 as a modulator of fibroblast growth factor responses. This work had employed microinjection of a SPRY2 octapeptide to mimic SPRY2 function and had shown that a peptide phosphorylated at the tyrosine equivalent to tyrosine 55 in full length SPRY2 blunted growth factor-induced ERK phosphorylation. Using a HIV-tat strategy to render the peptide cell permeable, we were indeed able to modify ERK responses following platelet activation, providing primary evidence that SPRY2 serves to modulate platelet responses. However, further work will be needed to fully define the functional role of SPRY2 within the complex context of MAPK signalling in platelet physiology. As such, the ERK pathway regulates store-mediated calcium entry in platelets, ${ }^{45}$ and is an important modulator of platelet integrin $\alpha$ IIb $\beta 3$ activation. ${ }^{46}$ In addition, ERK plays distinct roles, depending on the context. For example, agonist-induced MAPK activation regulates early but transient platelet granule secretion, while integrin-mediated MAPK activation facilitates late and sustained responses such as clot retraction. ${ }^{47}$

\section{Limitations}

In this study, we used an unbiased GWAS approach to identify genetic predictors of moderate to severe postoperative thrombocytopenia after CABG surgery. However, several limitations remain. First, because the identified SNP is a tagging marker in the intergenic region of LOC729479 I $S P R Y 2$, the observed associations could be due to changes in the regulation of gene expression or high LD with the true unidentified causal SNPs. Indeed, intergenic regions can be transcribed and the resulting long non-coding ribonucleic acids are known to perform several different functions, ranging from regulation of epigenetic modifications and gene expression to acting as scaffolds for protein signalling complexes. ${ }^{48}$ Therefore, future research to identify causal intergenic $\operatorname{SNP}(\mathrm{s})$ and to decipher their relationship to epigenetic modifications and/or expression of their neighbouring genes is important.

Second, based on current sample size and an incidence of moderate to severe post-operative thrombocytopenia of $39.6 \%$, an allele frequency of 0.22 as our top SNP (rs9574547), and a complete LD between SNP and causal variant, our power calculations show that our study has $73.4 \%$ power to detect a genotypic relative risk of 0.61 , which is equivalent to an OR of 0.44 . Although we used a relatively large cohort of cardiac surgery patients, our study is powered to detect only common variants with relatively large effects. Thus, we did not examine the possibility of rare genetic variants that influence a pronounced clinical phenotype.

Third, several non-genetic clinical and procedure-related factors are potentially associated with a higher risk for postoperative thrombocytopenia. ${ }^{3,4}$ However, given our study design and the relative size of the discovery and replication cohorts, we were not able to investigate effects of these factors on post-operative thrombocytopenia.

Fourth, of the 176 patients in the discovery cohort and the 76 patients in the replication cohort with moderate to severe post-operative thrombocytopenia, none developed heparininduced thrombocytopenia (HIT). However, per institutional protocol, routine testing for post-operative HIT is left to the discretion of the intensive care unit team. Nevertheless, as indicated by our previous studies, ${ }^{3,4}$ early-onset and persistent thrombocytopenia in CABG surgery patients is seldom caused by post-operative HIT and thus it is unlikely that we may have missed a diagnosis of post-operative HIT.

Fifth, our finding that SPRY2 modulates platelet ERK signalling and the critical influence of RTK signalling on platelet activation supports the intriguing hypothesis that the observed variation noted upstream of the SPRY2 gene modulates platelet function. However, the association of the identified SNP also with pre-operative platelet counts could suggest that variants of SPRY2 may take effect on platelet generation or maturation, a situation that may be further unmasked by peri-operative platelet loss and consumption. Indeed, a work by Yang et $\mathrm{al}^{49}$ suggests that SPRY1 may negatively regulate haematopoiesis. However, while a role of SPRY2 in thrombopoiesis cannot currently be excluded, we verified the association of rs9574547 with post-operative platelet count independent of the pre-operative platelet status by multivariable regression analysis. In addition, it is important to note that in our cohort-patients undergoing CABG surgery-pre-operative platelet counts cannot be taken as a true baseline/resting status. In fact, significant on-going platelet activation has been observed in these patients and linked to the underlying vasculopathy $y^{50-52}$ and to co-existing diseases such as hypertension ${ }^{53}$ or diabetes mellitus. ${ }^{54}$ In addition, we screened existing literature and studies that relied on available databases such as the UK Biobank (http://www.ukbiobank.ac.uk) and the GRASP (https://grasp.nhlbi.nih.gov/Search.aspx) database. We found that Astle et al observed no statistically significant association ( $p=0.22$ ) between a genetic variation in our top SNP, $r s 957454$, and (elevated) platelet counts in a large cohort of subjects with European ancestry. ${ }^{14}$ Hence, the findings of our study are more likely to reflect that SPRY2 itself or rs9574547 are linked to the cardiac surgical setting and/or disease aetiology rather than they are having a strong generalized effect on thrombogenesis. 
Similarly, we cannot currently exclude that, in absence of any platelet anomaly, endothelial or inflammatory SPRY2 targets drive the decline of platelet count after cardiac surgery. Our ex vivo platelet analysis clearly demonstrated that SPRY2 is present in platelets and regulated following activation. Therefore, our study provides the first evidence of SPRY2 involvement in modulating platelet functions, but more work is needed to define not only the role of SPRY2 but also of rs9574547 allele-specific differences for platelet responses and outcomes in cardiac surgery.

Finally, all of our study subjects were of European descent and, thus, our findings cannot be generalized to other ethnic groups.

In summary, we conducted a comprehensively designed GWAS in a cohort of patients at risk for moderate to severe post-operative thrombocytopenia after CABG surgery, and we identified and characterized a novel intergenic susceptibility locus for moderate to severe postoperative thrombocytopenia after CABG surgery with $\mathrm{CPB}$. The development of post-operative thrombocytopenia is associated with a significant risk of experiencing adverse outcomes such as stroke, ${ }^{4}$ AKI and increased mortality. ${ }^{3}$ Currently, it remains unknown what causes reduction of platelet numbers after cardiac surgery and how such thrombocytopenia is linked to the development of adverse outcomes. As a consequence, our data suggesting that modulated expression of the endogenous tyrosine kinase inhibitor SPRY2 is protective against the development of thrombocytopenia opens new opportunities to better understand the underlying pathophysiology and to develop pharmacological approaches to prevent post-operative thrombocytopenia. Notably, the on-going development of anti-platelet agents that target the tyrosine kinase pathway may provide novel therapeutic options to limit the extent of peri-operative platelet activation and the associated end-organ injury and mortality.

\section{What is known about this topic?}

- Thrombocytopenia after cardiac surgery is an independent predictor of stroke, acute kidney injury and death.

- However, baseline and clinical patient characteristics do not adequately account for the occurrence of postoperative thrombocytopenia.

\section{What does this paper add?}

- This study identified a genetic polymorphism in the intergenic region upstream of sprouty 2 (SPRY2) that is associated with decreased risk for developing postoperative thrombocytopenia after CABG surgery.

- This study also shows that SPRY2, which acts as an endogenous receptor tyrosine kinase inhibitor, is expressed in platelets and modulates ERK signalling during platelet activation.

- These findings support a role for SPRY2 in the modulation of platelet responses after cardiac surgery.
Funding

Funding for this study was provided by: the Duke Anesthesiology Developing Research Excellence in Anesthesia Management (DREAM) Award (to Dr. Kertai); the National Institutes of Health grants 1R56HL12689101 (to Dr. Karhausen), HL075273 and HL092071 (to Dr. Podgoreanu), HL096978, HL108280 and HL109971 (to Dr. Mathew), HL095987 (to Dr. Shah) and HL101621 (to Dr. Kraus); the American Heart Association grants 15SDG25080046 (to Dr. Karhausen), 9951185U (to Dr. Mathew) and 0120492U (to Dr. Podgoreanu); and a Duke School of Medicine Health Scholar award (to Dr. Karhausen). Logistics support was provided by CATHGEN. The authors are solely responsible for the design and conduct of this study, all study analyses and drafting and editing of the manuscript and its final contents.

\section{Conflict of Interest}

None.

\section{Acknowledgements}

The authors thank Jasmine Fowler, BS, for technical support, Dr. Gowthami M. Arepally, MD (Division of Hematology at Duke University Medical Center) for critical discussions and help with functional platelet testing, and Dr. Sara Galletti, PhD, for editing support. The members of the Duke Perioperative Genetics and Safety Outcomes (PEGASUS) Investigative Team are: Cooter $\mathrm{M}$, Daneshmand M, Funk B, Gaca JG, Ghadimi K, Ginsburg GS, Glower DD, Haney J, Hauser E, Karhausen J, Kertai MD, Laskowitz DT, Li YJ, Lodge AJ, Mathew JP, Milano CA, Moretti EW, Newman MF, Quinones QJ, Podgoreanu MV, Schroder J, Smith MP, Smith PK, Stafford-Smith M, Swaminathan $\mathrm{M}$, Waldron $\mathrm{NH}$ and Welsby IJ.

\section{References}

1 Paparella D, Yau TM, Young E. Cardiopulmonary bypass induced inflammation: pathophysiology and treatment. An update. Eur J Cardiothorac Surg 2002;21(02):232-244

2 Williamson DR, Lesur O, Tétrault JP, Nault V, Pilon D. Thrombocytopenia in the critically ill: prevalence, incidence, risk factors, and clinical outcomes. Can J Anaesth 2013;60(07):641-651

3 Kertai MD, Zhou S, Karhausen JA, et al. Platelet counts, acute kidney injury, and mortality after coronary artery bypass grafting surgery. Anesthesiology 2016;124(02):339-352

4 Karhausen JA, Smeltz AM, Akushevich I, et al. Platelet counts and postoperative stroke after coronary artery bypass grafting surgery. Anesth Analg 2017;125(04):1129-1139

5 Weerasinghe A, Taylor KM. The platelet in cardiopulmonary bypass. Ann Thorac Surg 1998;66(06):2145-2152

6 Lapchak PH, Kannan L, Ioannou A, et al. Platelets orchestrate remote tissue damage after mesenteric ischemia-reperfusion. Am J Physiol Gastrointest Liver Physiol 2012;302(08):G888-G897

7 Singbartl K, Forlow SB, Ley K. Platelet, but not endothelial, Pselectin is critical for neutrophil-mediated acute postischemic renal failure. FASEB J 2001;15(13):2337-2344

8 Mangano DT; Multicenter Study of Perioperative Ischemia Research Group. Aspirin and mortality from coronary bypass surgery. N Engl J Med 2002;347(17):1309-1317

9 Verma S, Goodman SG, Mehta SR, et al. Should dual antiplatelet therapy be used in patients following coronary artery bypass 
surgery? A meta-analysis of randomized controlled trials. BMC Surg 2015;15:112

10 Kunicki TJ, Williams SA, Nugent DJ. Genetic variants that affect platelet function. Curr Opin Hematol 2012;19(05):371-379

11 Petersen R, Lambourne JJ, Javierre BM, et al. Platelet function is modified by common sequence variation in megakaryocyte super enhancers. Nat Commun 2017;8:16058

12 Shameer K, Denny JC, Ding K, et al. A genome- and phenome-wide association study to identify genetic variants influencing platelet count and volume and their pleiotropic effects. Hum Genet 2014; 133(01):95-109

13 Johnson AD, Yanek LR, Chen MH, et al. Genome-wide metaanalyses identifies seven loci associated with platelet aggregation in response to agonists. Nat Genet 2010;42(07):608-613

14 Astle WJ, Elding H, Jiang T, et al. The allelic landscape of human blood cell trait variation and links to common complex disease. Cell 2016;167(05):1415-1429.e19

15 Eicher JD, Chami N, Kacprowski T, et al; Global Lipids Genetics Consortium; CARDIoGRAM Exome Consortium; Myocardial Infarction Genetics Consortium. Platelet-related variants identified by Exomechip meta-analysis in 157,293 individuals. Am J Hum Genet 2016;99(01):40-55

16 Morawski W, Sanak M, Cisowski M, et al. Prediction of the excessive perioperative bleeding in patients undergoing coronary artery bypass grafting: role of aspirin and platelet glycoprotein IIIa polymorphism. J Thorac Cardiovasc Surg 2005;130(03):791-796

17 Welsby IJ, Podgoreanu MV, Phillips-Bute B, et al; Perioperative Genetics and Safety Outcomes Study (PEGASUS) Investigative Team. Genetic factors contribute to bleeding after cardiac surgery. J Thromb Haemost 2005;3(06):1206-1212

18 Rinder CS, Mathew JP, Rinder HM, et al; Multicenter Study of Perioperative Ischemia Research Group. Platelet PlA2 polymorphism and platelet activation are associated with increased troponin I release after cardiopulmonary bypass. Anesthesiology 2002;97 (05):1118-1122

19 Muehlschlegel JD, Perry TE, Liu KY, et al. Polymorphism in the protease-activated receptor- 4 gene region associates with platelet activation and perioperative myocardial injury. Am J Hematol 2012;87(02):161-166

20 Little J, Higgins JP, Ioannidis JP, et al. Strengthening the reporting of genetic association studies (STREGA): an extension of the STROBE Statement. Hum Genet 2009;125(02):131-151

21 Kertai MD, Li YW, Li YJ, et al; Duke Perioperative Genetics and Safety Outcomes (PEGASUS) Investigative Team. G proteincoupled receptor kinase 5 gene polymorphisms are associated with postoperative atrial fibrillation after coronary artery bypass grafting in patients receiving $\beta$-blockers. Circ Cardiovasc Genet 2014;7(05):625-633

22 Kertai MD, Li YJ, Ji Y, et al; Duke Perioperative Genetics and Safety Outcomes (PEGASUS) Investigative Team. Genome-wide association study of new-onset atrial fibrillation after coronary artery bypass grafting surgery. Am Heart J 2015;170(03):580-90.e28

23 American Society of Anesthesiologists Task Force on Perioperative Blood Transfusion and Adjuvant Therapies. Practice guidelines for perioperative blood transfusion and adjuvant therapies: an updated report by the American Society of Anesthesiologists Task Force on Perioperative Blood Transfusion and Adjuvant Therapies. Anesthesiology 2006;105(01):198-208

24 Purcell S, Neale B, Todd-Brown K, et al. PLINK: a tool set for wholegenome association and population-based linkage analyses. Am J Hum Genet 2007;81(03):559-575

25 Price AL, Patterson NJ, Plenge RM, Weinblatt ME, Shadick NA, Reich D. Principal components analysis corrects for stratification in genome-wide association studies. Nat Genet 2006;38(08): 904-909

26 Marchini J, Howie B, Myers S, McVean G, Donnelly P. A new multipoint method for genome-wide association studies by imputation of genotypes. Nat Genet 2007;39(07):906-913
27 Hanafusa H, Torii S, Yasunaga T, Nishida E. Sprouty1 and Sprouty2 provide a control mechanism for the Ras/MAPK signalling pathway. Nat Cell Biol 2002;4(11):850-858

28 Comerford KM, Leonard MO, Karhausen J, Carey R, Colgan SP, Taylor CT. Small ubiquitin-related modifier-1 modification mediates resolution of CREB-dependent responses to hypoxia. Proc Natl Acad Sci U S A 2003;100(03):986-991

29 Schwarz G. Estimating the dimension of a model. Ann Stat 1978;6 (02):461-464

30 Pruim RJ, Welch RP, Sanna S, et al. LocusZoom: regional visualization of genome-wide association scan results. Bioinformatics 2010;26(18):2336-2337

31 Wei Y, Tejera P, Wang Z, et al. A missense genetic variant in LRRC16A/CARMIL1 improves acute respiratory distress syndrome survival by attenuating platelet count decline. Am J Respir Crit Care Med 2017;195(10):1353-1361

32 Chen MH, Yanek LR, Backman JD, et al. Exome-chip meta-analysis identifies association between variation in ANKRD26 and platelet aggregation. Platelets 2017. Doi: 10.1080/09537104.2017

33 Guy GR, Jackson RA, Yusoff P, Chow SY. Sprouty proteins: modified modulators, matchmakers or missing links? J Endocrinol 2009; 203(02):191-202

34 Lemmon MA, Schlessinger J. Cell signaling by receptor tyrosine kinases. Cell 2010;141(07):1117-1134

35 Lee RH, Bergmeier W. Platelet immunoreceptor tyrosine-based activation motif (ITAM) and hemITAM signaling and vascular integrity in inflammation and development. J Thromb Haemost 2016;14(04):645-654

36 Gould WR, Baxi SM, Schroeder R, et al. Gas6 receptors Axl, Sky and Mer enhance platelet activation and regulate thrombotic responses. J Thromb Haemost 2005;3(04):733-741

37 Boulaftali Y, Hess PR, Getz TM, et al. Platelet ITAM signaling is critical for vascular integrity in inflammation. J Clin Invest 2013; 123(02):908-916

38 Angelillo-Scherrer A, Burnier L, Flores N, et al. Role of Gas6 receptors in platelet signaling during thrombus stabilization and implications for antithrombotic therapy. J Clin Invest 2005; 115(02):237-246

39 Gratacap MP, Martin V, Valéra MC, et al. The new tyrosine-kinase inhibitor and anticancer drug dasatinib reversibly affects platelet activation in vitro and in vivo. Blood 2009;114(09):1884-1892

40 Metharom P, Berndt MC, Baker RI, Andrews RK. Current state and novel approaches of antiplatelet therapy. Arterioscler Thromb Vasc Biol 2015;35(06):1327-1338

41 Dütting S, Vögtle T, Morowski M, et al. Growth factor receptorbound protein 2 contributes to (hem)immunoreceptor tyrosinebased activation motif-mediated signaling in platelets. Circ Res 2014;114(03):444-453

42 Delehanty LL, Mogass M, Gonias SL, Racke FK, Johnstone B, Goldfarb AN. Stromal inhibition of megakaryocytic differentiation is associated with blockade of sustained Rap1 activation. Blood 2003;101(05):1744-1751

43 Edwin F, Anderson K, Patel TB. HECT domain-containing E3 ubiquitin ligase Nedd4 interacts with and ubiquitinates Sprouty2. J Biol Chem 2010;285(01):255-264

44 Nadeau RJ, Toher JL, Yang X, Kovalenko D, Friesel R. Regulation of Sprouty2 stability by mammalian Seven-in-Absentia homolog 2 . J Cell Biochem 2007;100(01):151-160

45 Rosado JA, Sage SO. Role of the ERK pathway in the activation of store-mediated calcium entry in human platelets. J Biol Chem 2001;276(19):15659-15665

46 Li Z, Xi X, Du X. A mitogen-activated protein kinase-dependent signaling pathway in the activation of platelet integrin alpha Ilbbeta3. J Biol Chem 2001;276(45):42226-42232

47 Flevaris P, Li Z, Zhang G, Zheng Y, Liu J, Du X. Two distinct roles of mitogen-activated protein kinases in platelets and a novel Rac1MAPK-dependent integrin outside-in retractile signaling pathway. Blood 2009;113(04):893-901 
48 Hangauer MJ, Vaughn IW, McManus MT. Pervasive transcription of the human genome produces thousands of previously unidentified long intergenic noncoding RNAs. PLoS Genet 2013;9 (06):e1003569

49 Yang X, Gong Y, Friesel R. Spry1 is expressed in hemangioblasts and negatively regulates primitive hematopoiesis and endothelial cell function. PLoS One 2011;6(04):e18374

50 Robless PA, Okonko D, Lintott P, Mansfield AO, Mikhailidis DP, Stansby GP. Increased platelet aggregation and activation in peripheral arterial disease. Eur J Vasc Endovasc Surg 2003;25(01):16-22

51 George R, Bhatt A, Narayani J, Thulaseedharan JV, Sivadasanpillai $\mathrm{H}$, Tharakan JA. Enhanced P-selectin expression on platelet-a marker of platelet activation, in young patients with angiographically proven coronary artery disease. Mol Cell Biochem 2016; 419(1-2):125-133

52 Tan KT, Tayebjee MH, Macfadyen RJ, Lip GY, Blann AD. Elevated platelet microparticles in stable coronary artery disease are unrelated to disease severity or to indices of inflammation. Platelets 2005;16(06):368-371

53 Gkaliagkousi E, Passacquale G, Douma S, Zamboulis C, Ferro A. Platelet activation in essential hypertension: implications for antiplatelet treatment. Am J Hypertens 2010;23(03):229-236

54 Ferroni P, Basili S, Falco A, Davì G. Platelet activation in type 2 diabetes mellitus. J Thromb Haemost 2004;2(08):1282-1291 